\title{
Una nueva zona de arte rupestre al aire libre en el NW: la península de Barbanza
}

\author{
Ángel Concheiro Coello \\ LOLA Gil AGRA ${ }^{1}$
}

\section{INTRODUCCIÓN}

Este artículo tiene un doble objetivo: por una parte dar a conocer una nueva zona de dispersión de grabados rupestres al aire libre; por otra acercarnos, no tanto a su significado, perspectiva, como veremos más adelante, sumamente problemática, sino a su lógica espacial y territorial, de modo que podamos extraer implicaciones sobre las formas de vida y organización social de las comunidades que los realizaron y utilizaron.

Este tipo de estudios, que relacionan los yacimientos con el espacio en que se integran, valorando siempre las estaciones de grabados como un elemento construido en el paisaje con claras connotaciones de caracterizador territorial, tienen en el NW una tradición limitada, vinculada, generalmente, a las corrientes teóricas derivadas de la Arqueología Espacial, bien sea en su vertiente neoarqueológica (PEÑA y REY 1993) o con matices "post-procesuales" (BRADLEY et al. 1993; VILLOCH 1993; FILGUEIRA y RODRIGUEZ 1994).

La Península de Barbanza era tradicionalmente una zona poco valorada por los estudiosos de este tipo de manifestaciones prehistóricas. La causa fundamental radicaba en la reducida cantidad de estaciones conocidas, existiendo zonas, como la mitad septentrional, que presentaban auténticos vacios. No obstante, el desarrollo de trabajos de prospección intensiva (CONCHEIRO 1989), así como hallazgos causales reali-

1 Rúa de Galicia, núm. 51, Noia, A Coruña. 
zados en fechas recientes han certificado lo erróneo de esta propuesta, si bien muchos de estos datos son todavía inéditos (REY y SoTO); GIL 1993). En este sentido, gran parte de las evidencias que manejaremos en nuestro trabajo son fruto del proyecto de prospección intensiva llevado a cabo en los meses invernales de 1989 y 1990, dirigido por uno de los autores (CONCHEIRO, A. ${ }^{2}$ ), enfocado a la búsqueda y localización de aquellos yacimientos protohistóricos que, por carecer de presencia monumental en el paisaje, no habian sido convenientemente valorados y permanecian infrarrepresentados en las catalogaciones y cartas arqueológicas al uso (Lopez y Bouza 1929; Calo 1986; Agrafoxo 1987).

\section{DESCRIPCIÓN GEOGRÁFICA}

La Península de Barbanza se encuentra situada en el litoral $W$ de Galicia, concretamente al Norte de las Rías Baixas (fig. 1). Geomorfológicamente sus $416 \mathrm{~km}$ cuadrados de extensión están definidos por la Sierra de Barbanza, una cadena montañosa de más de $6 \mathrm{~km}$ de largo que recorre longitudinalmente, en sentido NE-SW, gran parte de la península. Se trata de un bloque macizo y poco articulado, coronado por un conjunto de valles interiores, cubetas, penillanuras y cumbres que llegan a superar los 600 m.s.n.m.

Los escarpes, muy pronunciados y abruptos, dan paso a las plataformas litorales, franjas de altitud inferior a los 200 m.s.n.m., en cuyo relieve predominan las formas suaves. La plataforma litoral $\mathrm{S}$, abierta a la ría de Arousa, se muestra más desenvuelta que la $\mathrm{N}$, bien sea a través de los cauces de los ríos (Coroño) o de las unidades geográficas menores (Peninsula de O Chazo).

Geológicamente en la península conviven materiales de distinto tipo y origen, desigualmente repartidos. El que tiene una mayor representación es el granito de dos micas de grano medio-grueso, de tipo Barbanza, mayoritario en todo el sector central. Otras rocas presentes son los esquistos y diversos materiales metamórficos que conforman la fosa blastomilonítica. Menos representados, pero no por ello de menor interés, son los depósitos de origen cuaternario, constituidos por materiales de origen sedimentario y localizados fundamentalmente en las zonas litorales.

2 Este proyecto fue subvencionado por la Dirección Xeral do Patrimonio Histórico e Documental da Xunta de Galicia. 
Climatológicamente habría que incluir a la Península dentro del dominio Oceánico Húmedo, caracterizado por una reducida oscilación térmica anual y pluviosidad acentuada con un máximo invernal, aunque con acusada tendencia a la aridez estival. De todos modos, la presencia y orientación de la Sierra favorece la existencia de una cierta variabilidad climática, no muy acusada, reflejada en el aumento proporcional de los índices de pluviosidad con respecto a la altitud, y en la existencia de temperaturas más altas en aquellas zonas abiertas al aire cálido de procedencia oceánica.

\section{PLANTEAMIENTOS METODOLÓGICOS}

Los criterios metodológicos utilizados en el trabajo de campo que aqui se presenta se integran en la práctica conocida como Prospección Intensiva. A grandes rasgos, ésta se caracteriza por el rastreo a pie, intensivo y sistemático, de la superficie en busca de restos arqueológicos que, debido a sus características intrínsecas, no presentan estructuras artificiales monumentales fácilmente reconocibles sobre el terreno.

La lógica interna del propio método de trabajo requiere que éste se centre sobre "unidades de paisaje significativas" (CRIADO et al. 1991), ello, unido a la gran extensión de la Península de Barbanza motivó la necesidad de seleccionar una serie de áreas ${ }^{3}$ en las que los trabajos realizados adquirian una coherencia que de otro modo solamente podria alcanzarse con la utilización de unos recursos, técnicos y humanos, totalmente ajenos a nuestro proyecto.

De esta forma, sin rechazar la posibilidad de que en un futuro se den a conocer un mayor número de evidencias arqueológicas en la zona objeto de estudio, pensamos que el registro arqueológico es lo suficientemente representativo para permitir un análisis del tipo que aquí se propone.

\section{INVENTARIO DE ESTACIONES}

\section{A Picota}

Localización: A Picota, Miñortos, Porto do Son.

3 Concheiro, A., "Prospección Intensiva en la Península de Barbanza: del Epipaleolítico a la Edad del Bronce", Memoria inédita depositada en la Dirección Xeral do Patrimonio Histórico e Documental. Xunta de Galicia. 
Emplazamiento: Media ladera de una vertiente que domina un pequeño valle transversal a la línea de costa.

Descripción: La estación consta de una única roca en la que se representan: una combinación circular formada por 4 radios concéntricos, cazoleta central con radio en prolongación exterior y final en curva; a esta figura se asocian dos círculos sencillos con cazoleta central y una cazoleta: mitad inferior de lo que podria ser una combinación circular con radio en prolongación exterior; mitad superior de una combinación circular formada por tres anillos concéntricos y radio prolongado interiormente inscribiendo 4 cazoletas; tres cazoletas dispersas y otros trazos rectilíneos.

\section{Outeiro de Malda}

Localización: Valcunqueiro, Goiáns, Porto do Son.

Emplazamiento: Media ladera sobre la cabecera de un pequeño valle transversal a la línea de la costa.

Descripción: La estación consta de dos grupos de grabados que se sitúan sobre un afloramiento granítico a ras de suelo y con una ligera inclinación hacia el SW. (fig. 6.3).

Grupo 1: Una combinación circular formada por dos círculos concéntricos y cazoleta interior. Once «herraduras". Seis serpentiformes. Dos círculos sencillos. Veinticuatro cazoletas.

Grupo 2: Un diseño geométrico con serpentiforme inscrito. Un círculo sencillo con cazoleta inscrita. Cuatro huellas de ungulado. Dos «herraduras". Un alfabetiforme. Nueve cazoletas.

Bibliografía: GIL 1993.

\section{Monte de Eiravedra}

Localización: Eiravedra, Goiáns, Porto do Son.

Emplazamiento: Sobre un afloramiento a modo de pequeño "outeiro" en la plataforma litoral.

Descripción: 1 cruciforme, 16 cazoletas (fig. 6.1).

Bibliografía: GIL 1993.

\section{Augapedra 1}

Localización: Castelo, Noal, Porto do Son.

Emplazamiento: Sobre un afloramiento rocoso que destaca sobre el entorno, predominantemente llano, de la plataforma litoral.

Descripción: 1 "pia» de la que sale un canal, 21 cazoletas.

Bibliografía: GIL 1993. 


\section{Augapedra 2}

Localización: Castelo, Noal, Porto do Son.

Emplazamiento: $100 \mathrm{~m}$ al $\mathrm{N}$ de la estación núm. 4, sobre una floramiento de características semejantes.

Descripción: 2 cazoletas.

Bibliografía: GIL 1993.

\section{Braña das Pozas}

Localización: Porto do Son, Noal, Porto do Son.

Emplazamiento: Se sitúa en la ladera NW del monte Dordo, a una altitud de $200 \mathrm{~m}$ y a medio camino entre el pie de monte y la cumbre. A $125 \mathrm{~m}$ en dirección SW tiene su nacimiento un pequeño curso de agua de carácter estacional.

Descripción: Los grabados se disponen en la cara SW de una roca de forma hemicilíndrica inclinada en el sentido de la pendiente, conformando un panel vertical de $4,10 \times 1,60 \mathrm{~m}$, de superficie lisa y regular. El conjunto está formado por 11 representaciones de zoomorfos que, en posición alineada, ocupan longitudinalmente el panel. Resulta llamativa la presencia de un cérvido macho dotado de cornamenta ramificada (fig. 5.1).

Bibliografía: GIL 1993; GIL y CONCHEIRO 1993.

\section{Laxe da Sartaña}

Localización: Cadarnoxo, Queiruga, Porto do Son.

Emplazamiento: Se sitúa a media ladera del primer escalón montañoso, dominando la plataforma litoral.

Descripción: Las representaciones se disponen sobre una roca de $30 \times 16,90 \mathrm{~m}$ que aflora a ras de suelo. En su superficie se observan tanto grabados antiguos como otros modernos superpuestos, que en muchos casos llegan a dañar seriamente a aquéllos. Por razones obvias sólo nos ocuparemos de los primeros. Los motivos aparecen concentrados en dos grupos o paneles.

Grupo 1: Veinte zoomorfos, posiblemente cérvidos, en manada. Una combinación circular formada por tres círculos concéntricos, radio curvo en prolongación y siete cazoletas inscritas. Una combinación circular formada por seis círculos concéntricos y radio inscrito. Una escena de monta (jinete con équido). Tres trazos paralelos. Un grupo de cuatro cazoletas.

Grupo 2: Cinco zoomorfos, probablemente cérvidos, en manada. Un círculo simple (incompleto). Líneas erráticas y trazos paralelos.

Bibliografía: REY y Soto (A). 


\section{Pedra das Cabras}

Localización: Figueirido, Palmeira, Ribeira.

Emplazamiento: Se sitúa sobre un rellano o escalón al pie de las primeras estribaciones de la sierra. Muy próximo a un arroyo.

Descripción: La estación se compone de una única roca grabada, de forma prácticamente hemicilíndrica. En su cara SW se representan, alineadas, dos figuras de zoomorfos, dotados de cuerpos voluminosos y extremidades realizadas con tres trazos, sumamente reducidas (fig. 5.3).

Bibliografia: PeÑa y VÁzQUeZ 1979; AgRafoxo 1987.

\section{Os Petóns do Castro}

Localización: Loxo de Abaixo, Cures, Boiro.

Emplazamiento: Se sitúa en un escalón que domina el valle del rio Coroño, bajo el escarpe $S$ de la sierra.

Descripción: La estación se compone de seis grupos de motivos, todos, excepto uno (grupo 6), dispuestos en la misma roca, de gran superficie, que aflora prácticamente a ras de suelo (fig. 6.2).

Grupo 1: Combinación circular formada por tres anillos concéntricos con trece cazoletas inscritas; de su centro parte un radio que se bifurca en dos líneas curvas. Una combinación circular.

Grupo 2: Tres combinaciones circulares formadas por dos círculos concéntricos, con sendos radios que encierran, respectivamente, seis, cuatro y siete cazoletas. Una combinación circular simple con radio y cuatro cazoletas inscritas.

Grupo 3: Un alfabetiforme.

Grupo 4: Una combinación circular de tres anillos abiertos y radio en prolongación.

Grupo 5: Dos combinaciones circulares con once y nueve cazoletas inscritas y radio en prolongación. Combinación circular simple con cinco cazoletas inscritas. Cinco cazoletas dispersas.

Grupo 6: Combinación circular simple con seis cazoletas inscritas y radio exterior en prolongación con extremo bifurcado.

Bibliografía: Agrafoxo 1987; GIL 1993.

10. Pedra da Bouza

Localización: Seán, Abanqueiro, Boiro.

Emplazamiento: La estación se sitúa en la parte superior de una suave loma, en la ruptura de pendiente que se orienta hacia el SE.

Descripción: Una combinación de dos círculos concéntricos y cazo- 
leta central, con radio en prolongación. Una combinación de dos círculos concéntricos, cazoleta central y radio; el círculo exterior, abierto, se une al motivo 1 por un radio en prolongación. Combinación de tres círculos concéntricos y cazoleta central; del círculo exterior parten dos anteniformes. Dos círculos simples con cazoleta central. Círculo simple con cazoleta central. Círculo simple con radio en prolongación. Tres cazoletas dispersas.

Bibliografía: GIL 1993.

\section{Pedra da Craba}

Localización: Cubeliño, Cespón, Boiro.

Emplazamiento: La estación se sitúa a media ladera, en el primer escalón de la plataforma litoral.

Descripción: Los grabados fueron ejecutados en la parte más elevada de un afloramiento acusadamente inclinado hacia el SE. Grupo 1: dos zoomorfos. Grupo 2: conjunto de nueve cazoletas (fig. 5.2).

Bibliografia: LÓpez BouzA 1927; Agrafoxo 1987; GlL 1993.

\section{Fontán 1}

Localización: Cubeliño, Cespón, Boiro.

Emplazamiento: En una zona de media ladera que conforma la cabecera de un pequeño valle, donde tiene su nacimiento un arroyo cuyo cauce es transversal a las tierras bajas de la plataforma litoral.

Descripción: Grupo 1: Combinación circular constituida por dos círculos concéntricos abiertos, cazoleta central y radio en prolongación. Motivo en "U». Dos semicirculos concéntricos. Cazoleta.

Grupo 2: Circulo simple con tres cazoletas inscritas y figura compleja asociada. Cruciforme. Alfabetiforme. Ocho cazoletas dispersas.

Grupo 3: Tres motivos formados por líneas curvas de aspecto serpentiforme. Seis cazoletas.

Bibliografia: GIL 1993.

\section{Fontán 2}

Localización: Cubeliño, Cespón, Boiro.

Emplazamiento: Se sitúa $30 \mathrm{~m}$ al NW de la estación núm. 12 por lo que comparte sus características de emplazamiento.

Descripción: Cuatro combinaciones circulares complejas con cazoleta central.

Bibliografía: GIL 1993. 


\section{Fontán 3}

Localización: Cubeliño, Cespón, Boiro.

Emplazamiento: Se sitúa $20 \mathrm{~m}$ al $\mathrm{W}$ de la estación núm. 13 por lo que comparte las características de emplazamiento con los dos casos anteriores. radio.

Descripción: Grupo 1: Combinación circular con cazoleta central y

Grupo 2: Conjunto de cazoletas.

Grupo 3: Líneas erráticas y motivos geométrico-esquemáticos.

Bibliografía: GIL 1993.

\section{Brión}

Localización: Brión, Abanqueiro, Boiro.

Emplazamiento: En la base del escalón que forma la transición entre las tierras bajas de la plataforma litoral y las primeras elevaciones.

Descripción: Grupo de cuatro cazoletas.

Bibliografía: GıL 1993.

\section{Monte Furado 1}

Localización: Vilariño, Cespón, Boiro.

Emplazamiento: Media ladera en la cabecera de un pequeño valle.

Descripción: Combinación circular compleja formada por dos círculos concéntricos con cazoleta interior. Un zoomorfo. Otros trazos inidentificables.

Bibliografía: Inédito.

\section{Monte Furado 2}

Localización: Vilariño, Cespón, Boiro.

Emplazamiento: Media ladera en la cabecera de un pequeño valle.

Descripción: Grupo 1: Tres zoomorfos, dos de ellos cérvidos machos dotados de cornamenta ramiforme. Dos combinaciones circulares complejas con cazoleta interior y radio en prolongación.

Grupo 2: Cuatro zoomorfos, todos ellos cérvidos machos dotados de cornamenta desarrollada. Otros motivos inidentificables y trazos imprecisos.

Grupo 3: Una combinación circular con cazoletas inscritas: Un motivo geométrico. Una cazoleta aislada.

Bibliografía: Inédito. 


\section{Axeitos}

Localización: Axeitos, Oleiros, Ribeira.

Emplazamiento: Media ladera en suave caída hacia un valle interior.

Descripción: Cuatro zoomorfos y una cazoleta.

Observaciones: Esta estación, situada $50 \mathrm{~m}$ al Sur del dolmen de Axeitos, ha desaparecido, quedando constancia de su existencia gracias al estudio en ella realizado por Cuevillas y Bouza Brey en 1927.

Bibliografía: LÓPEZ y Bouza 1927; Agrafoxo 1986.

\section{Outeiro Redondo}

Localización: Sabuceda, Cures, Boiro.

Emplazamiento: Suave loma que domina visualmente un área de relieve deprimido (braña).

Descripción: Dos cazoletas.

Bibliografía: Inédito.

\section{Rio Barbanza}

Localización: Sabuceda, Cures, Boiro.

Emplazamiento: Fondo de valle.

Descripción: Grupo de dos cazoletas.

Bibliografía: Inédito.

\section{CONSIDERACIONES FORMALES: TEMÁTICA Y ESTILO}

La temática desarrollada en las estaciones de la Península de $\mathrm{O}$ Barbanza sigue las pautas del denominado "grupo galaico": Cazoletas/puntos, combinaciones circulares, figuras geométricas, zoomorfos, huellas de animales... Únicamente están ausentes las representaciones de armas y se observa un claro predominio de los grabados de carácter abstracto sobre aquellos de tipo subnaturalista.

Dentro de los motivos de naturaleza abstracta el tipo más abundante es el de las cazoletas/puntos, que aparecen acompañadas por otros motivos o formando un grupo propio de representación. Están presentes en 19 de las 20 estaciones contempladas.

El segundo tipo de carácter abstracto cuantitativamente más representado es el de las combinaciones circulares, que manifiestan distinto grado de complejidad, aunque el modelo más común es aquel formado por doble/triple anillo concéntrico con cazoleta central. Está presente en 10 estaciones. 
Otros motivos englobados dentro del calificativo "abstracto" son las figuras cuadrangulares, los diseños geométricos, los ondulados, las "herraduras", las pías, los canales y las líneas erráticas. Aparecen representados de modo muy minoritario entre 1 y 3 estaciones (fig. 6).

Dentro del grupo de figuras subnaturalistas destacan las representaciones de zoomorfos, presentes en ocho estaciones. Aunque siempre resulte problemático, se han podido distinguir cérvidos en seis estaciones, los más numerosos; los équidos y los serpentiformes aparecen únicamente en una estación. Otros motivos, como las huellas de animales, que pueden considerarse como una representación metonímica, y la figura humana, en este caso asociada a un équido en una escena de monta, están presentes también en una sola estación.

En este tipo de grabados se aprecian una serie de aspectos de indole compositivo que sería necesario resaltar. El primero se refiere a la superficie en que fueron realizados, pues en dos casos, tres si añadimos el desaparecido de Axeitos, la representación está dispuesta en paneles verticales, con lo que su notoriedad en el paisaje queda sumamente destacada. El segundo radica en el desarrollo de escenas de carácter naturalista, generalmente la representación del acto sexual, o grupos de animales en marcha, en un caso acompañados de un équido con jinete. En estas escenas de manadas en movimiento las figuras se disponen ascendiendo en el sentido de la ladera, por lo que podemos hablar de una interpretación real de la topografía en la que se emplazan las estaciones (GIL y ConCHEIRO 1993).

Respecto a las consideraciones estilísticas que se pueden extraer del análisis de los grabados, nos centraremos únicamente, y como resulta obvio, en las representaciones subnaturalistas. Aunque gran parte de ellas resultan inclasificables, podemos establecer en base a la distinta formalización plástica de las figuras dos tipos básicos:

Tipo 1: Formado por figuras de carácter geométrico, realizadas mediante tres surcos que siluetean un cuerpo estilizado de aspecto rectangular, dentro del que se exageran ciertas partes anatómicas, como las cornamentas. Manifiesta un cierto "aire de familia" con otras estaciones del área septentrional de las Rias Baixas (EIROA y REY 1938; BonILLA et al. 1993). Este tipo está presente en las estaciones de Braña das Pozas, Laxe da Sartaña, Monte Furado 2 y Pedra da Craba (figs. 5.1 y 5.2).

Tipo 2: Constituido por figuras de animales de contornos achaparrados y redondeados, con cuello y extremidades poco desarrolladas. A este grupo pertenecen las estaciones de Pedra das Cabras y la desa- 
parecida de Axeitos. Paralelos próximos se encuentran en la estación de Os Ballotes, Vilagacía (PEÑA y VÁzquez 1979) (fig. 5.3).

Con respecto a la distribución de uno y otro tipo, observamos que el primero se dispersa por toda la península, mientras el segundo se ciñe exclusivamente a la orilla Sur, certificando que se trata de una manifestación sumamente original característica de la ría de Arousa.

\section{DISTRIBUCIÓN Y EMPLAZAMIENTO}

Las estaciones se distribuyen a lo largo del territorio de un modo relativamente disperso, sin mostrar concentraciones excesivamente importantes, a excepción del sector NE de la península de O Chazo, en el Ayuntamiento de Boiro, con ocho estaciones, y en la orla litoral NE, dentro del término municipal de Porto do Son, con seis estaciones (fig. 1).

A partir de una escala de análisis espacial que comprende la totalidad de la Península, se observa una predilección de las estacones por emplazarse en las tierras de menor altitud. De hecho, y a excepción de las núm. 19 (Outeiro Redondo) y 20 (Río Barbanza), localizadas en lo alto de la Sierra, a más de $500 \mathrm{~m}$ de altitud, todas se sitúan por debajo de la curva de nivel de $200 \mathrm{~m}$.

Esta vinculación con las tierras de menor altitud se hace más evidente si atendemos a su relación con las distintas unidades fisiográficas. De las 20 estaciones reconocidas, 14 se encuentran sobre la plataforma litoral o escalones adyacentes, dos (núm. 2 y núm. 6, Outeiro da Malda y Braña das Pozas) lo hacen en un punto intermedio, de transición entre las tierras bajas y los escarpes; y, como hemos visto anteriormente, únicamente dos en las tierras altas de la Sierra.

Existe pues una clara regularidad, matizable por supuesto, que asocia las estaciones de grabados rupestres con las tierras bajas próximas al litoral. Esta relación ya ha sido constatada en áreas geográficas de características semejantes, como la Península de O Morrazo (PEÑa y ReY 1993).

Aunque requiere un análisis más pormenorizado y un número mayor de evidencias, resulta hasta cierto punto lógico hablar de una "exclusividad espacial» según la cual los petroglifos se emplazan de modo mayoritario en los terrenos de valle que desarrollan hacia el interior la plataforma litoral, ocupando sus vertientes hasta una cota no excesivamente elevada y que, en ningún caso, supera los $200 \mathrm{~m}$. En contraposición, las tierras que forman los escarpes de la Sierra están absolutamente vacías de representaciones de este tipo, mientras su presencia en el planalto resulta muy minoritaria. 
Esta concentración de las estaciones en áreas de características físicas determinadas puede reflejar, a escala regional, la propia distribución de las comunidades que los realizaron, como se intuye a partir de los mapas de localización de yacimientos, en los que se observan los mayores indicios de ocupación doméstica en esas mismas áreas inmediatas al litoral (Concheiro, A.; Peña y Rey 1993; Gil 1993).

En todo caso, a la hora de extraer consecuencias socio-culturales de carácter menos general es necesario recurrir a análisis más detallados sobre el patrón de emplazamiento, no ya de los petroglifos grosso modo, sino de los distintos tipos de motivos representados, conscientes de que este tipo de manifestación, que comunica contenidos de carácter ideacional, es muy posible que englobe realidades complejas y diferenciables. En ese sentido, el objetivo de este estudio no es intentar descodificar un mensaje indescifrable, sino desarrollar una lectura del petroglifo como un hecho cultural y consciente que, por sus propias caracteristicas, objetiviza y dota de significados sociales a determinadas partes del paisaje.

Este tipo de análisis, por otra parte, sólo tiene sentido si se realiza sobre zonas geográficas poco extensas, bien definidas a nivel físico y con un número suficiente de evidencias que faciliten el establecimiento de diferentes niveles de relación espacial. Para ello seleccionamos dos áreas, situadas en márgenes opuestos de la Península que, pensamos, se corresponden con los presupuestos anteriormente comentados: el sector costero septentrional de Porto do Son, a orillas de la ría de Muros y Noia, y la península de 0 Chazo-valle del río Coroño, volcada dentro de la ria de Arousa.

1. Sector costero septentrional. Porto do Son (fig. 2). Esta unidad está formada por una plataforma litoral de relieve suavemente ondulado, articulada en dos valles que la amplían hacia el interior (val de Nebra, val de Miñortos) y que dan paso, primero de manera gradual, por medio de ligeros escalones dominados por pequeños cerros, y luego más acusadamente, a los escarpes que delimitan las tierras altas de la Sierra.

En ella se localizan, hasta el momento, un total de seis estaciones: A Picota (núm. 1), Outeiro da Malda (núm. 2), Eiravedra (núm. 3), Augapedra 1 (núm. 4), Augapedra 2 (núm. 5) y Braña das Pozas (núm. 6). Como ya hemos comentado anteriormente, las estaciones parecen vincularse, de uno u otro modo, con las tierras de menor altitud, si bien es posible apreciar diferencias significativas en la distribución de los distintos motivos. De hecho, se observa un claro contraste entre las estaciones con grabados formalmente sencillos (grupos de cazoletas) y aquellas formadas por motivos y composiciones de carácter complejo (zoomorfos, combinaciones circulares...). Las primeras se disponen en zonas de valle, de 
tierras pesadas y muy próximos al litoral, con pendientes siempre inferiores al $10 \%$, emplazándose sobre pequeños outeiros o afloramientos que destacan visualmente sobre el entorno inmediato. Las segundas lo hacen a media ladera, generalmente en las vertientes de pequeños valles que conducen a los escarpes de la sierra.

2. Peninsula de O Chazo-val do rio Coroño (fig. 3). Ostenta unas condiciones físicas muy parecidas a las de la zona anterior, quizás enriquecidas por la presencia de pequeñas subunidades fisiográficas de personalidad acusada ( $O$ Chazo). En ella se encuentran un total de nueve estaciones: Os Petóns do Castro (núm. 9), Pedra da Bouza (núm. 10), Pedra da Craba (núm. 11), Fontán 1 (núm. 12), Fontán 2 (núm. 13), Fontán 3 (núm. 14), Brión (núm. 15), Monte Furado 1 (núm. 16), Monte Furado 2 (núm. 17). También aquí se observa una distribución selectiva de las estaciones de acuerdo con el tipo de motivos que representan. Asi, se aprecia que la única estación compuesta exclusivamente por cazoletas (Brión, núm. 15) se sitúa en el fondo de un valle inmediato a la costa. El resto, aunque en algún caso se localizan también cerca del litoral (Pedra da Craba, núm. 11), se emplazan, como se constató en el área anterior, en zonas de ladera, a medio camino entre las tierras bajas del litoral y valles adyacentes, y los escarpes de la Sierra o de los macizos montañosos subsidiarios. Esto resulta muy significativo en el sector NE, donde existe una sensible concentración de estaciones (núms. 11, 12, 13, 14, 16 y 17) que se distribuyen formando una orla en profundidad alrededor de un pequeño valle transversal a la línea de costa (Fig. 4).

La consecuencia más destacable de este análisis es la constatación de una serie de pautas que definen un patrón de distribución diferencial para los dos grandes grupos de representación: las estaciones formadas exclusivamente por cazoletas y los conjuntos de grabados complejos, sean de carácter abstracto o subnaturalista.

Este patrón dual no se ciñe a su distinta relación con la orografía, sino que se observan características semejantes en sus relaciones con otros elementos del medio natural. Éstas son especialmente significativas en el caso de las estaciones con grabados complejos. En general, y esto parece ser una constante, las estaciones de este tipo están próximas a zonas húmedas o cursos de agua, bien en forma de pequeños ríos (núms. 14, 15 y 16), fuentes (núm. 7) o brañas (núm. 18). Con respecto a sus condiciones de visibilidad, éstas, aparentemente, son más variables: o disfrutan de amplias panorámicas (núms. 2, 6), o la percepción del entorno resulta restringida (núms. 13, 14, 15 y 16). En todos los casos, sin embargo, la superficie grabada se orienta hacia zonas concretas del paisaje, generalmente las vertientes medias de pequeños valles 
transversales a la costa que actúan y actuaron como áreas naturales de paso, o hacia áreas de relieve ligeramente cóncavo donde se encuentran superficies encharcadas de modo semiperenne.

Debemos intentar comprender, por último, si este patrón de distribución responde a una lógica social o, por contra, se trata de un fenómeno puramente aleatorio. El mapa de distribución de yacimientos contemporáneos revela que, en gran parte, es la propia naturaleza de los petroglifos la que condiciona sus relaciones con los otros yacimientos, a semejanza de lo que acontecía con el medio natural.

Observamos una clara dicotomía:

Por un lado, las estaciones formadas exclusivamente por cazoletas se sitúan o en las inmediaciones de aquellas áreas donde aparecen indicios de actividad doméstica persistente, con las que compartirian lo que convencionalmente se ha denominado "área de explotación preferencial" (RUIz ZAPATERo 1988) o en las cercanias de necrópolis tumulares, situadas en terrenos de elevada altitud.

Por otro, las estaciones con grabados complejos se localizan en puntos del paisaje en los que sería difícil desempeñar una actividad doméstica continuada, por lo que es complicado detectar cualquier indicio arqueológico que no sean los propios grabados, pudiendo éstos vincularse más al tránsito entre diferentes áreas de explotación o zonas de explotación temporal o extensiva.

Esta asociación se clarifica con la observación de un mapa de distribución al que se le ha aplicado la técnica del "vecino más próximo" (figs. 2 y 3). En él se aprecia que el yacimiento más cercano a un petroglifo exclusivamente de cazoletas es, o bien otra estación del mismo tipo, o bien un área con un fuerte componente de actividad antrópica, sea doméstica o funeraria; mientras que para las estaciones complejas el yacimiento más próximo es siempre otra representación del mismo tipo, no siendo extraño que no aparezcan directamente relacionadas con ninguna otra clase de evidencia.

\section{CONCLUSIONES; HACIA LA FORMULACIÓN DE UN MODELO TERRITORIAL}

Pensamos que existen suficientes puntos de apoyo para hablar de una clara asociación entre determinados tipos de representaciones y áreas concretas del paisaje. Las estaciones formalmente más simples se localizan a distinta cota, bien en tierras bajas cercanas a la costa, bien en las 
zonas altas de la Sierra, pero siempre en clara relación, hasta el punto de compartir un espacio común, con las mayores concentraciones de actividad doméstica y/o funeraria. En algunos casos, como ocurre con las estaciones núms. 19 y 20 , casi inmediatas a los túmulos de Outeiro Redondo y Arca da Barbanza, o con las estaciones núms. 4 y 5 , muy próximas al asentamiento de Cabeiro, de cronología Calcolítico-Campaniforme, la relación espacial resulta tan evidente que podria hablarse de un cespacio común" para estos tipos de manifestaciones arqueológicas. Éste no es un hecho en absoluto nuevo en Galicia, ya que se constata en otras zonas como la Península del Morrazo (PENA y REY 1993), la más meridional de las situadas en las Rías Baixas, la comarca lucense de O Incio (FILgUeIRAs y Rodriguez 1994) y el área de As Rozas (NILLOCH 1993), en el interior de la provincia de Pontevedra. Fuera del NW, esta misma asociación ha sido documentada en la región escocesa de Argyll (BRADLEY 1991).

Los petroglifos complejos, por su parte, siguen un patrón de distribución que podriamos denominar "en profundidad". Las estaciones se disponen de modo escalonado, a lo largo de las vertientes de pequeños valles que ascienden desde la plataforma litoral hacia las tierras del interior, de altimetría considerablemente más elevada. Sus concentraciones más importantes, caso del sector NE de la Península de $\mathrm{O}$ Chazo (fig. 4), se localizan precisamente en estas vertientes que facilitan el tránsito entre las tierras litorales y los planaltos de la Sierra.

La relación de estas estaciones con las áreas de explotación de los asentamientos contemporáneos alude a una posible funcionalidad territorial. La dimensión liminar de los petroglifos de cazoletas, rodeando necrópolis o pequeños ecosistemas de naturaleza diversificada, la vinculación de gran parte de las estaciones a puntos del paisaje que remarcan líneas de tránsito entre dominios geográficos distintos, a lo largo de "Corredores" ecológicamente variados (BRADLEY et al. 1993) y las propias caracteristicas de los poblados de esta cronología, generalmente pequeñas aldeas de carácter no permanente, nos remite al modelo teórico de una sociedad dotada, todavía, de un importante componente móvil. Su patrón de asentamiento estaría parcialmente condicionado por unas prácticas de obtención de alimentos dentro de las que, sin negar las labores agrícolas, jugarían un papel destacado las estrategias derivadas de la explotación directa de los recursos naturales (HAYDEN 1990; BRADLEY 1993).

Existen razones suficientes para pensar los petroglifos como parte de un determinado sistema de apropiación del espacio. Sus características de distribución y emplazamiento, a todas luces conscientemente selectivas, parecen responder a un patrón estable que los asocia bien a zonas de intensa actividad humana, bien a áreas naturales de paso, bien a otros 
terrenos potencialmente interesantes (brañas, fuentes y pequeños cursos de agua). En este sentido resulta significativo señalar que las estaciones compuestas por zoomorfos, que en varios casos representan manadas de animales en marcha ascendente, y las representaciones de huellas de animales formando sendas, se sitúan siempre en áreas naturales de paso, a media ladera, utilizadas todavia hoy en sus desplazamientos por el ganado mostrenco semisalvaje. El hecho de que esta relación se establezca, en muchos casos, desde una óptica de dominio visual, nos lleva a considerar a estas estaciones como delimitadores de los espacios concretos de explotación y de las áreas de tránsito que estructuran un territorio concebido de manera lineal.

\section{ABSTRACT}

A new area with open air rock carvings in the Iberian NW; the peninsula of Barbanza.

Traditionally the research on Galician rock art had focused in the area around Campolameiro (Pontevedra) where the biggest number of sites was reported, whereas other zones to the North were almost unknown in that respect. In this paper we deal with the new finds of petroglyphs in the Barbanza, where intensive survey has multiplied the number of this tipe of sites. The usual motifs are abstract (mainly concentric circles and cupmarks) but also semischematic with representations of deer. The distribution of the art is more dense in the coastal lowlands, though a number of sites is reported in the passes leading into the high sierra and in this area itself. As to the relationship with the roughly contemporary settlements (IIIrd millenium BC), we have observed a definite pattern: cupmarks are sharing the same space with the more densely inhabited areas down to the coast and also they are found near to the mounds in the high plateau; animal representations and more complex abstract motifs (concentric circles with or without radius) are sited along small valleys or ridges that link the lowlands and the upper reaches of the Sierra. All this patterning would point out to the rôle of petrogliphs as a mechanism for defining the most resourceful or symbolic places and the paths leading into them.

\section{BIBLIOGRAFIA}

Agrafoxo Pérez, X. (1987): Prehistoria e Arqueoloxia da Terra da Barbanza. Santiago. Bonilla Rodriguez, A.; Parga Castro, A.; Torres Álvarez, A. (1993): Nueva Zona de Grabados Rupestres en Galicia: prospección intensiva del ayuntamiento de Rianxo. Inédito. 
Bradley, R. (1991): "Rock Art and the Perception of Landscape", Cambridge Archaeological Journal, 1. Cambridge.

- (1993): Altering the Earth. Edimburgo.

Bradley, R.; Criado Boado, F., y Fábregas Valcarce, R. (1993): Petroglifos en el Paisaje: nuevas perspectivas sobre el arte rupestre gallego. Minius 2-3. (En prensa).

Calo Lourido, F. (1986): Castro de Baroña. Santiago.

Concheiro Coello, A. (A): Prospección en la Península de Barbanza. La evolución del hábitat entre el Epipaleolítico y la Edad del Bronce. Arqueoloxia-Informes 3 (En prensa).

Criado BoAdo, F., et al. (1991): Arqueología del Paisaje: el Area Bocelo-Furelos entre los tiempos Paleoliticos y Medievales. Santiago.

EIROA, J.J., y Rey CAStiñeiRas, J. (1986): Guía de los Petroglifos de Muros. Muros.

Filgueiras Rey, A., y Rodriguez Fernández, T. (1994): Túmulos y Petroglifos. La Construcción de un Espacio Funerario. Aproximación a sus Implicaciones Simbólicas. (En prensa).

GIL AgRA, L. (1993): Una Aproximación a la Organización del Espacio en la Prehistoria Reciente: La Peninsula do Barbanza. Trab. Inv. $3 .^{\circ}$ ciclo. (Inédito).

Gil Agra, L., y Concheiro Coello, A. (1993): A Estación de Grabados Rupestres de "Braña das Pozas" (Porto do Son, A Coruña). Minius 2-3. (En Prensa).

HAYDEN, B. (1990): "Nimrods, Piscators, Pluckers and Planters: the emergence of food production", Antropol. Archaeol., 9.

lópez Cuevillas, F., y Bouza Brey, F. (1927): Prehistoria e Folklore da Barbanza. A Coruña.

Peña Santos, A., y Rey Garcia, J.M. (1993): El Espacio de la Representación. El Arte Rupestre Gallego desde una Perspectiva Territorial. Pontevedra 10.

Peña Santos, A., y VázQuez Varela, J.M. (1979): Los Petroglifos Gallegos. Sada, A Coruña.

Rey Castiñeiras, J., y Soto Barreiro, M. ${ }^{a}$ J. (A): A Laxe da Sartaña (Porto do Son, A Coruña). Memoria de investigación. Dirección Xeral de Patrimonio Histórico e Documental. Xunta de Galicia. Inédito.

Ruiz Zapatero, G., y Burillo Mozota, F. (1988): “Metodologia para la Investigación en Arqueología Territorial", Munibe, sup. 6. San Sebastián.

VILLOCH VÁzoUEZ, V. (1993): La Configuración del Espacio en las Sociedades Prehistóricas de Galicia: Análisis del Emplazamiento de Túmulos y Petroglifos. Trab. Inv. $3 .^{\circ}$ ciclo. (Inédito). 

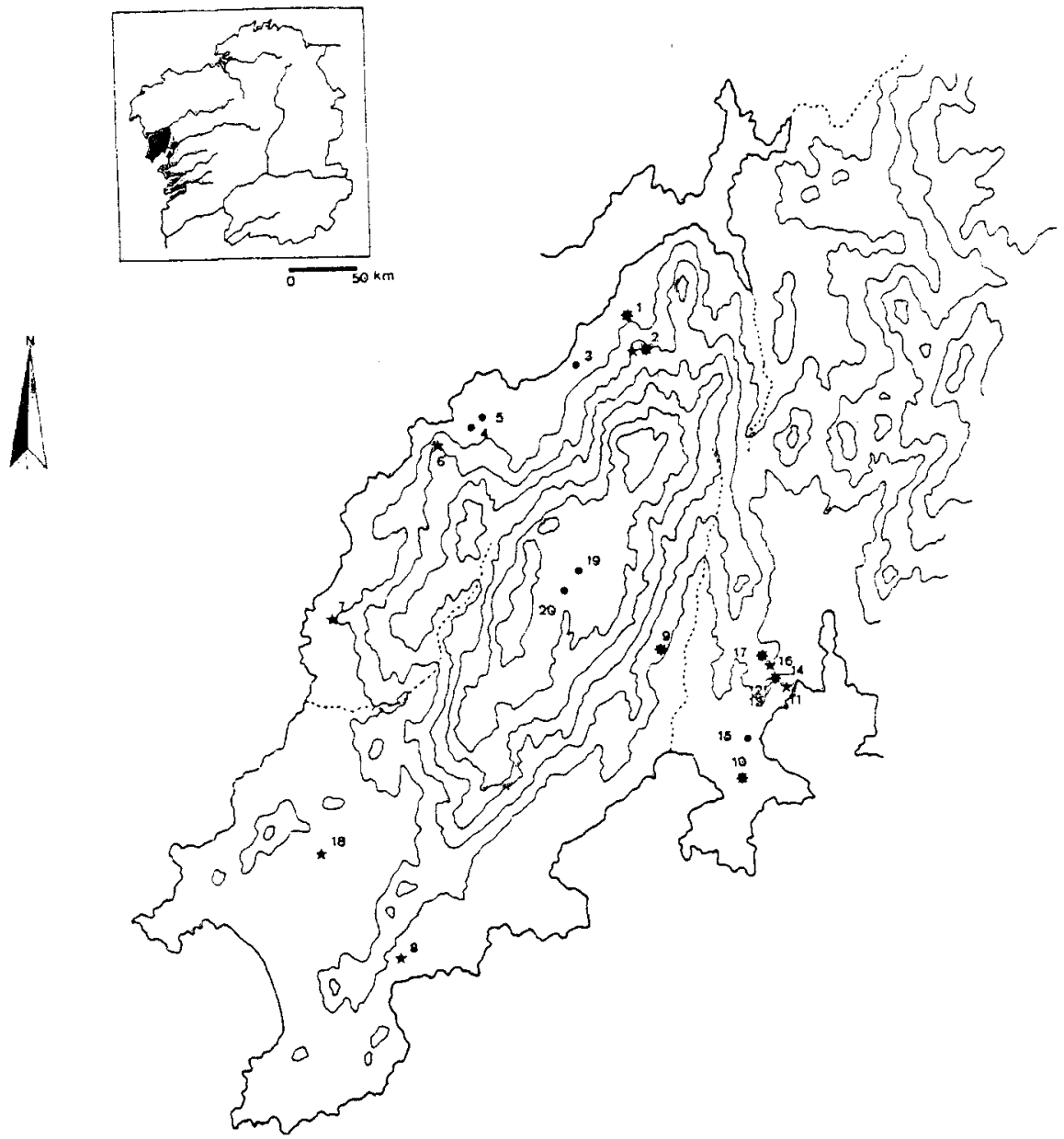

0 $10 \mathrm{~km}$

- stmples

* aestractos complejos

7 subnaturalistas

Fig. 1. La Peninsula de Barbanza, A Coruña. Localización de las estaciones. 


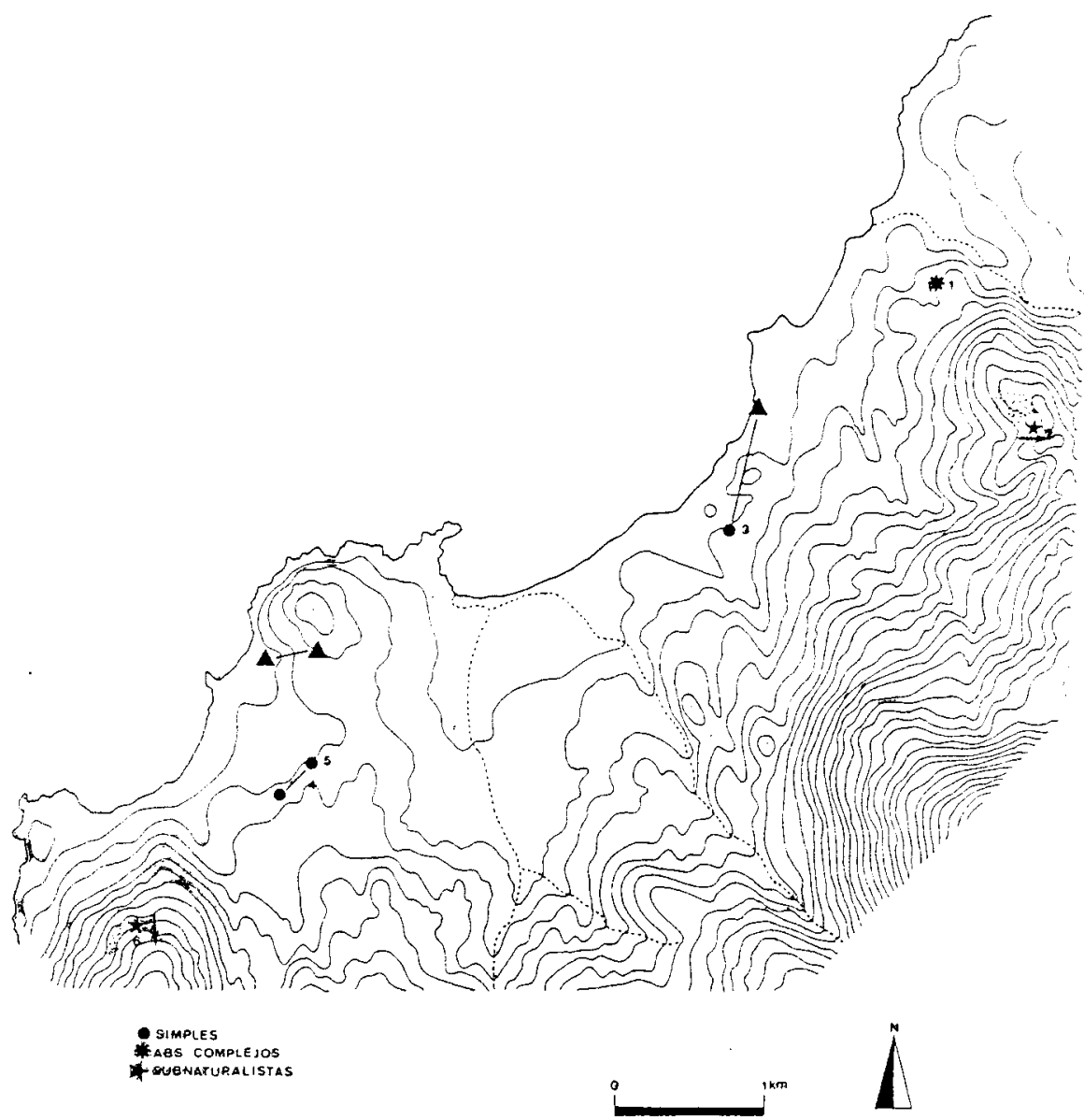

Fig. 2. Sector costero de Porto do Son. Estaciones de grabados rupestres al aire libre y su relación con los asentamientos campaniformes.

Linea: Distancia entre yacimientos más próximos.

Flecha: Orientación de los zoomorfos y huellas.

Sombreado: Braña. 


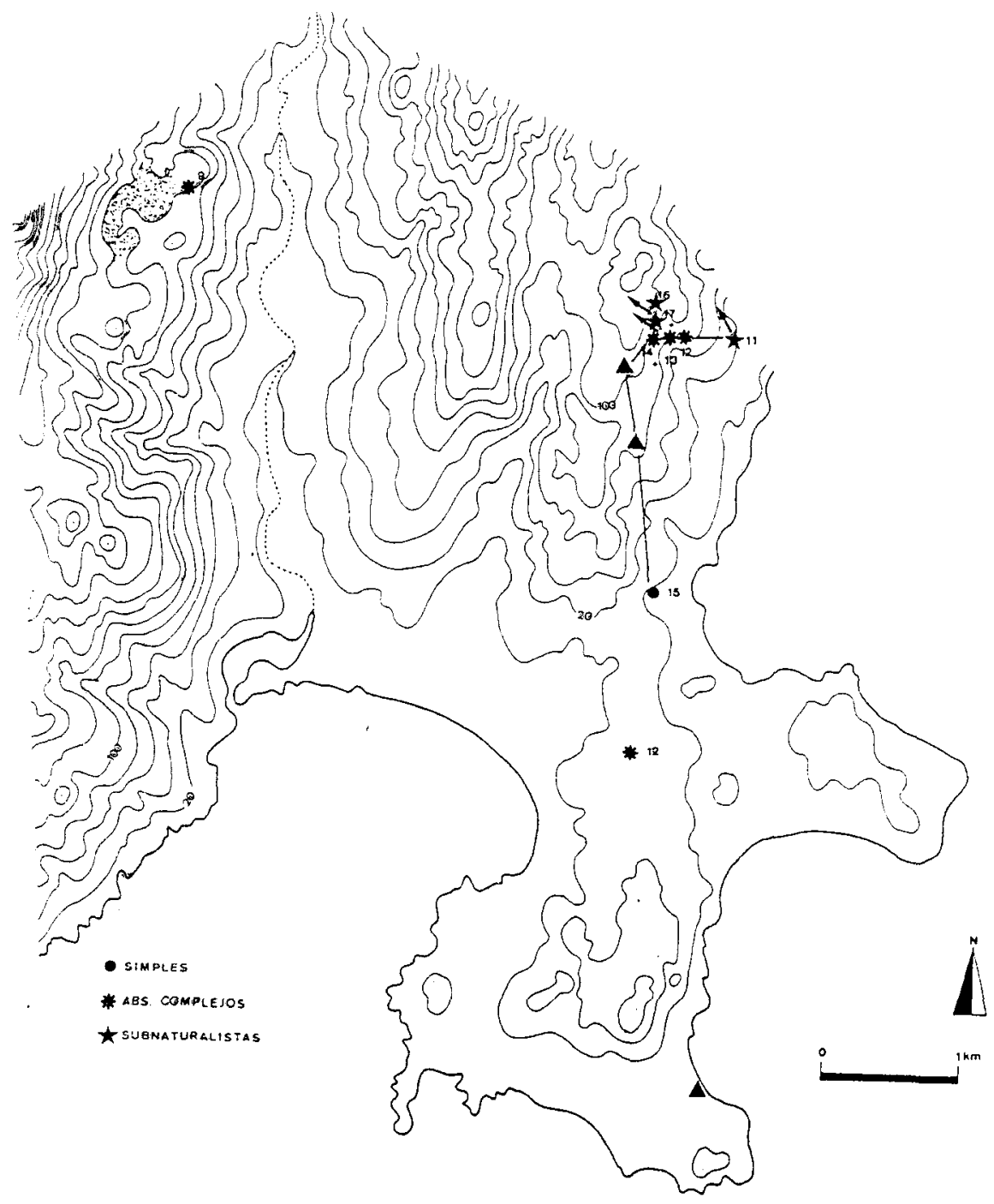

Fig. 3. Peninsula de O Chazo y valle del rio Coroño. Estaciones de grabados rupestres al aire libre y su relación con asentamientos campaniformes.

Línea: Distancia entre yacimientos más próximos.

Flecha: Orientación de los zoomorfos y huellas.

Sombreado: Braña. 


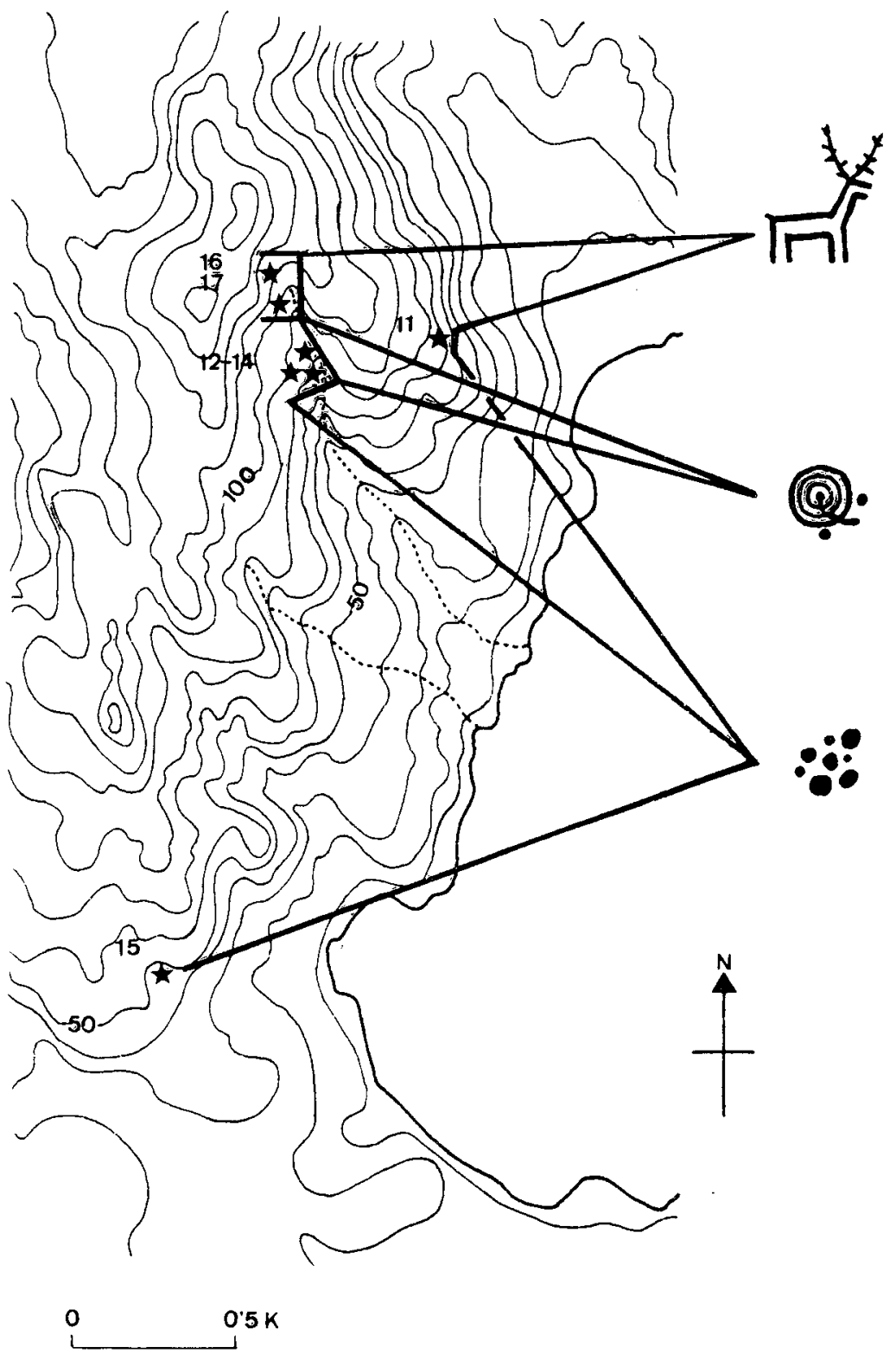

Fig. 4. Sector NE. Península de O Chazo. Emplazamiento puntual de las estaciones. 

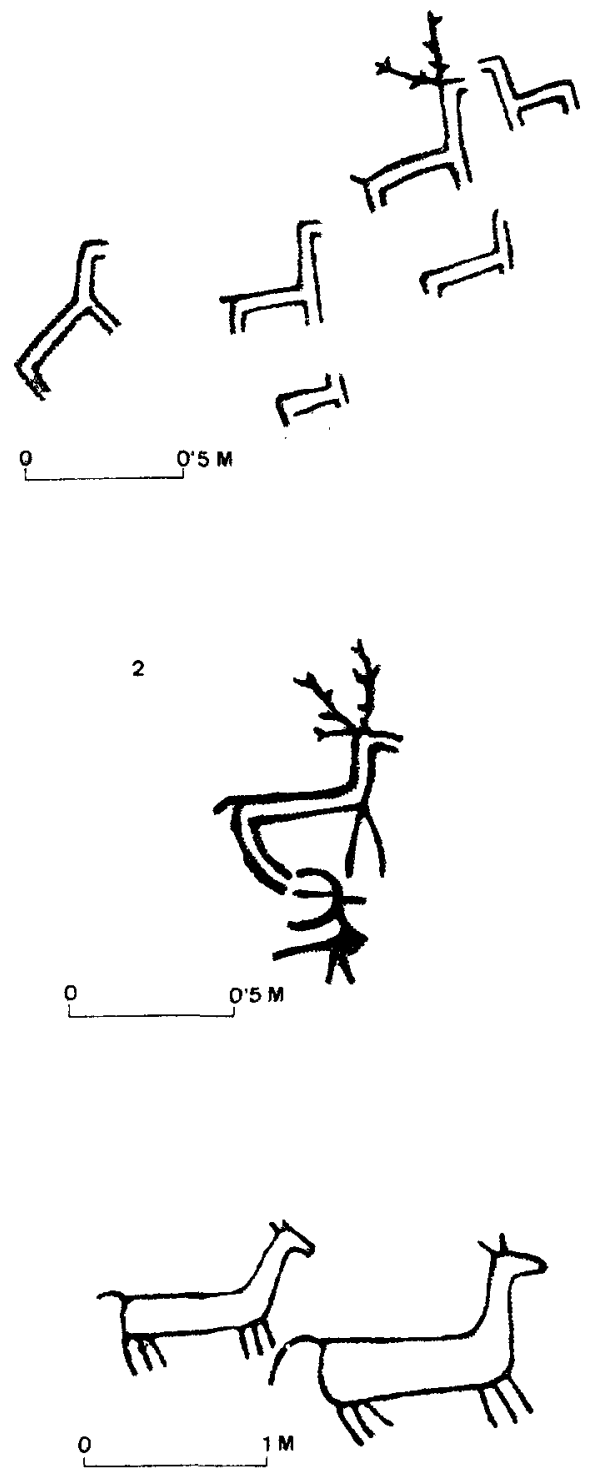

Fig. 5. Representaciones subnaturalistas: 1. Braña das Pozas (detalle). 2. Pedra da Cabra. 3. Pedra das Cabras. 

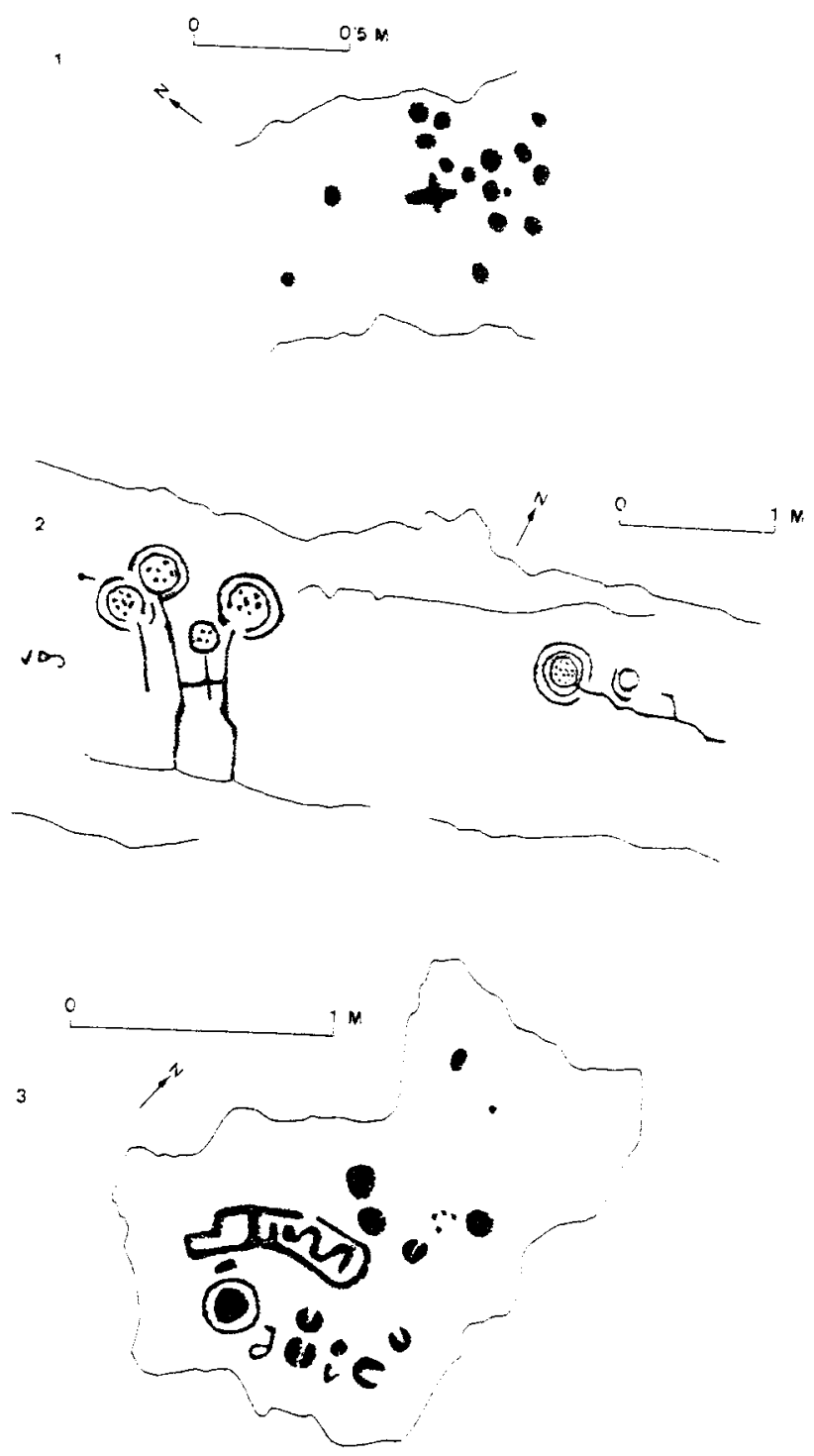

Fig. 6. Estaciones con motivos abstractos: 1. Eiravedra. 2. Os Petóns do Castro (grupos 1, 2, 3). 3. Outeiro da Malda (grupo 2). 